\title{
Organization of animal-assisted therapy: From voluntary activities to work of professionals
}

\author{
Marika Lotko \\ Department of Welfare and Social Work, Rīga Stradinsš University, Riga, Latvia
}

\begin{abstract}
Humankind has always known about the usefulness in integration of animals for the achievement of such routine goals as hunting, household and territory protection, transportation, scouting foreign territories and materials, and overcoming physical limitations. Animals are also highly valued as agents of socialisation resulting in the integration of various species in the work of various professions. Social work also increasingly makes use of professional activities involving animals while working with clients. Terms such as therapy animals, animal-assisted therapy, animal-assisted activities, and animal-assisted education are becoming more relevant in the daily work of social workers, physiotherapists, occupational therapists, psychotherapists and other professionals. Animals are more and more often found in hospitals, schools, care facilities, prisons and other settings. Animal-assisted therapy is a general term including various activities, causing difficulty to understand and differentiate between the types of therapy performed with various animal species, which creates the need to study the experience in ensuring work with therapy animals in various settings. The results obtained in this study reflect the experiences working with therapy animals of various professionals including the goal of integrating therapy animals in professional activity, the goal of the activity and the steps thereof, target groups and the expected results.
\end{abstract}

\section{Introduction}

\subsection{Human-animal interaction}

Human-animal studies (HAS) is one of the most recent multidisciplinary studies where the study object is focused on the human-animal relationship and interaction, and the use of animals in therapy contexts, and which discusses the ways to understand animals. HAS strives towards understanding animals in the context of human society and culture, the use of animals in literature and art, the relationship between companion animals and their human families, the use of animals as symbols in religion and language, and the use thereof in agriculture and biomedical research [1].

\subsection{Research on interaction with animals}

Research describes the benefits of interacting with animals in detail and its impact on human well-being and decision-making.

Fine (2006) declares that the presence of animals increases social support and considers the animal as a catalyst, promoting interaction between humans. Animals can improve human life, especially in lonely humans. An animal is always available, ensures comfort, relaxation and entertainment. One of the primary benefits of animal company may be associated with 
decrease in human suffering, depression and loneliness. Animals strongly support their owners and serve as a buffer against failures [2].

Norton (2010) notes that, allowing teenagers in care centres to keep animals, increases their sense of responsibility and the ability to care for others [3].

Melson et. al. (2009) compared child interaction with living and robotised dogs and found that children exercised petting and scratching actions, spoke more and asked questions more frequently to a living than a robotised dog [4]. Espinoza et. al. (2011) studied the interaction between a humanoid robot and children who have recently started to walk at a paediatrics unit [5].

Banks et. al. (2008) researched treating loneliness with AIBO robot dogs in seniors in long-term care facilities. He found that one of the ways to decrease loneliness in people in long-term care facilities is the use of animal-assisted therapy. The use of robotised dogs became especially popular in Japan and other Western countries where having a dog is difficult due to lifestyle choices [6].

Simmons et. al. (2007) highlighted that identification of animal abuse can help to identify domestic violence [7].

Thompson et. al.(2006) identified the importance of noting that homeless youths with pets will choose pet-friendly social services that allow their presence in relevant institutions [8].

The mentioned animal interaction studies present the attempts to understand individual benefits, as well as the effect on decision-making upon choosing social services, and the promotion of human well-being by means of modern technologies if interaction with live animals is impossible. Due to cultural differences, the cultural context can have an impact on the context and substance of the animal interactions.

\subsection{Application of terminology}

When describing work with different animal species, scientific publications use different terminology and refer to the species as well as to the professional and animal-therapy goal. The literature includes such terms as animal-therapy, animal-assisted therapy, animal-assisted activities, animal-assisted education, animal-facilitated therapy, dog therapy, equine-assisted therapy, dolphin-assisted therapy, equine-facilitated therapy, etc. Each of these terms may have significant differences, which is highlighted in the tasks intended for the animal, the specific nature of animal training, the length of interaction activities with the client and involvement in working with large client flow.

According to De Mello (2012), therapy animals are specifically trained to promote mental, physical and emotional care for clients suffering from various problems and diseases. The animals used in animal-assisted therapy are called therapy animals and are chosen because of their delicate temper and the ability to help clients. They include dogs, cats, rabbits, horses, birds, guinea pigs and dolphins [1].

According to Fine (2006), animal-assisted therapy and activities can include various ways of involving animals, for instance, indirect observation in the presence of the animal, direct observation or interaction [2].

One of the models, defining animal activities in this context, was proposed by Ensminger (2010). He divided animal-assisted interference into three groups: 1) animal-assisted therapy; 2) animal-assisted activities; and 3) animal-assisted education [9].

Upon defining animal-assisted interference, McCardle includes several activities where the animal is a participant with the aim to assist the client/patient. Such activities include animal interaction and are associated with relaxation involving the four senses - smell, touch, auditory and visual. The client/patient can see, smell, pet and talk to the animal and its owner, listen to the animal and hear the owner's interpretation of the animal's behaviour [10]. 
Marcus (2012) defines animal-assisted therapy as a goal-oriented interference wherein the animals meet specific criteria and are integrated in the process of treating clients with appropriate social problems and health conditions. The involvement of animals in such treatment process is intended for specific and measurable target improvement [11].

According to Lindquist (2014), specific animal-assisted therapy goal and tasks are not planned individually per client/patient; visit activities are spontaneous, their duration depends on the individual case, and documenting the interaction is not mandatory. Animal-assisted activities are less structured and offer human-animal contact for the purposes of recreation, education or enjoyment [12].

The discrepancies in the use of terminology require the need to study the identification of animal-therapy activities, their goal and substance within different institutions/settings.

\subsection{Required animal qualities}

While working with clients/patients, it is expected of the animal to demonstrate specific behaviour, carry out the intended tasks and possess certain qualities.

A crucial minimum in this regard is good animal health. No manifestation of behaviour that could be harmful to the client (jumping on/off, biting, avoidance or obvious aggression), obedience, the animal should be living with the owner or have an established relationship with $\mathrm{him} /$ her over a specific period of time, and must be comfortable in various environments. The animal must feel comfortable being picked up and surrounded by a group of people, be able to handle stressful situations, enjoy patient interaction, must not manifest signs of stress, fear, aggression, or shyness, and should not avoid human touch (Mongillo et. al., 2015) [13].

Evaluation by The American Kennel Club (AKC) includes a Canine Good Citizen Test (CGC) [14], which can be used to evaluate canine behaviour and temper. The AKC CGC was first introduced in 1989 to ensure that man's best companion is a good member of our society. The CGC test includes such criteria as friendliness towards strangers, allowing petting by strangers, allowing care and check-up by strangers, walking on a loose leash, walking through a crowd of people, sitting and staying on command, coming when called, polite behaviour in the presence of other dogs, giving in to panic when confused, and the retention of trained skills when walked by strangers.

\section{Material and methods}

Semi-structured interviews were used to conduct the study with therapy animal handlers $(n=15)$ in different settings in states of West Virginia and New York, USA. The owners of the therapy animals were working at the educational institutions (two of whom work with children with behavioural issues, others work with students), NGO who work with people with disabilities and children up to 10 years old who learn reading. The studies in the aforementioned institutions used therapy canines and equines, whereas one institution used farm animals while working with clients/patients.

The partially structured interviews were carried out in 2017.

The goal of the study was to investigate the work of professionals who integrate therapy animals in their professional activities, and to reveal the goal and content of the work and activities with the involved therapy animals.

During the partially structured interviews the following work aspects were studied: the use of terminology upon defining therapy animals and the appropriateness of the activities thereof, professional experience in working with therapy animals, the goal and specific nature of working with therapy animals, financing of provided activities and animal-keeping costs, 
the necessary therapy animal and handler certification, limitations for working with therapy animals, and institutional requirements for keeping therapy animals.

\section{Results}

Response analysis indicates various goals for integrating therapy animals in different activities. The results from respondents working in educational institutions indicate that canines are expected to spend time with students/pupils and the staff, allow them to be petted, be playful and just be there for people. There is no therapy work involved.

"The dog is an addition for the purpose of creating a friendly and relaxed environment..."

"Students do not always admit to being home-sick, and a dog can help greatly with that.

The pressure is linked with drug and alcohol use."

It should be noted that all canines living in educational institutions are labelled as therapy dogs. A volunteer for an NGO integrates canines into various activities:

"We have Reading to the Dogs Programs in 4 libraries, teams in the United Hospital Centre, nursing homes for the elderly as well as other medical institutions, a reading program in several elementary schools, and we participate in community activities (children fairs, introduction to dog breeds, etc.)."

Another respondent indicated that spending time with animals is part of the therapy programme:

"Our main goal is helping children recover and learn through nature and nurture - being outdoors with animals, implementing patient therapy approach where children try to deal with past trauma or a mental disorder."

One respondent who works with horses stated:

"We have clients with autism, Alzheimer's disease, rare genetic disorders, etc. We will continue to provide more equine-assisted education. We are increasing the amount of activities with horses, and not just riding but also working with horses, which means groundwork instead of working while horseback riding."

Compiling respondents' answers revealed that therapy animals help with various functions: therapy, recreational and educational activities. Therapy aspects are especially highlighted in equine-assisted therapy, while according to respondents' experience canine-assisted therapy is mainly oriented towards recreation and spending time together.

“... We do various activities, and all of them are volunteer activities. I am the main point of contact for people interested in therapy dog visits to their institution."

In regards to documenting work involving therapy animals, professionals ensure work reports, which is the case for NGOs providing various canine activities, and fill out documentation to identify the client's progress, which is the case for NGOs ensuring equine-assisted therapy.

In regards to the coverage of animal keeping-related expenses (feeding, vet visits, etc.), such expenses are only covered by the institution if the therapy animal is "owned" by this institution (identified in two cases regarding farm animals and equine-therapy animals); the rest of the expenses are personally covered by the animal handler.

Animal and human training and certification for the purpose of provision of animal therapy is received in various institutions. 
"The canines must attend basic training required for socialising, which makes the canines happy and keeps them safe for the society and themselves, the handler must be able to handle a canine, and both the handler and the canine must be well-kept and social."

"Canines of our employees must pass the Good Citizenship Test."

In one educational institution, all interviewees had to pass special training and certification in order to become therapy canine owners. Therapy canines owned by the interviewees have previously had a training programme provided by NGOs ensuring service dog training, but for some reason had failed to become a service dog. These dogs were afterwards waiting for a home, and 7 of them (in 2017) became therapy dogs at the mentioned educational institution.

"We have never had a problem with the placement of dogs. We have people waiting for a dog. I think that's because the dogs spend a lot of time with people. People know them and there is always someone waiting for a dog in case it will not become a service dog. If we need to discharge a dog to become a pet, we ask for a donation in return. We usually ask for at least 600\$, but that's not enough to cover training, maintenance and other costs. We offer our service and emotional support dogs free of charge."

Several therapy dog handlers have certified themselves and their dogs at the Alliance of Therapy Dogs, which is a part of the International Registry of Certified Therapy Dog Teams [15].

"Both of us passed the testing and registration process. I have my member card which I carry with me, and Brenda [the therapy dog] has its own, and it has a badge as well which she carries on her collar every time we go on a therapy visit. Every year we have to register and confirm we are in line with all of their requirements, required medical procedures and vaccinations, and then they send us a renewed card. They repeat the testing process after any changes in the status, such as injuries, diseases, or any incidents."

Institution professionals ensuring equine-assisted therapy are certified at the Professional Association of Therapeutic Horsemanship International (PATH).

"I am a PATH-registered instructor, too."

Farm animals, however, require no certification. In order to bring a therapy animal to an institution, it can request compliance with specific terms, which is the case in hospitals, for instance. The respondents were asked if their represented institution has any specific terms or guidelines regulating the presence of therapy animals, respectively.

"We are members of PATH, which has its own animal behaviour and care guidelines, and we follow them closely. Members learn about them in training."

One of the respondents whose represented institution ensures interaction with farm animals, stated:

"Every task description states that it is a nature-based programme, and that it includes animals, and because it is a large part of what we do, we want people to feel comfortable, so we try to integrate that in our work as much as possible. It is included in our manual and every task description."

Professionals working with therapy dogs, however, have no specific institutional/ organisational guidelines or requirements. Only representative of an educational institution stated:

"Only one of the faculties has developed individual guidelines for the presence of therapy dogs." 
The planning of activities integrating therapy animals should consider the following limitations: a person's fear of a specific species of animal, allergies, and cultural differences.

"We never force children to interact with animals if they are afraid or don't want to do it. We will definitely try to work on it little by little. There have been many children, who are afraid of animals at the beginning, so we start working with them later on, especially with dogs and horses. We've also had children with severe allergies. In one case, we discovered the child really cannot spend time with animals. In most of these cases, we try to find appropriate medical means to ensure the opportunity to establish some contact with animals.

"Many of our international students are afraid of the dog. In some countries, children do not have pets, so I keep it [the dog] on a short leash. I can usually tell the students keep away at the foyer. I know there are two students at the faculty who are afraid of dogs so I do not take my dog there, besides one of the students is severely allergic. Even if the dog is hypo-allergenic. We have an employee who says she is allergic but has never shown any symptoms. Some people may still be allergic. But if I'm aware of a potential problem, I never take my dog near the person. When I'm in a crowd, I usually look around to see the people's reaction."

Analysis of responses, regarding the benefits of integrating therapy animals into their professional activities reveals that a sense of safety is increasing and the person is able to open up mentally and talk about personal issues. It is easier to establish contact by starting a conversation about the animal. If a client does not want to make eye contact with the professional, they can have it with the animal; therapy animals give a sense of freedom for clients with disabilities; clients can repeat activities that were carried out in an institution with a therapy animal at home, which promote confidence in one's own abilities, etc.

"Often when a visit is held at one common room for all interested clients, we find that one of the benefits of interacting with us is that people interact with each other as well. That's something they wouldn't otherwise do."

"I'm not sure if I can describe the benefits. A child that was initially petrified of a horse was lying on its back after six sessions. There was another person that was unable to walk, but horseback riding provided a sense of freedom because it simulates walking."

"I had a child with domestic issues and we held sessions over Skype because the parents were far away. We decided to involve a dog, because the child kept leaving. It was clear that the child could not then leave the session because it would make the dog unsafe and run away. The child never tried to leave the session afterwards, as he was worried about the dog. When he felt uncomfortable and avoided eye contact with me or his mom, he could communicate with the dog which was a nice little break for him. Such interaction would be impossible without the presence of an animal."

There is no uniformity of opinion between respondents working with therapy animals when it comes to the use of terminology to describe animal activities and work goals. The respondents mostly identified support and entertainment, but no therapy functions for dogs. A clear boundary between therapy and entertainment activities was provided by a volunteer at an NGO that provides various activities and does community work.

"We call our visits animal-assisted therapy. There are two types of visits: one type of visits are relaxed one-to-one visits, and the other involves a team working with a therapist to ensure patient therapy to solve specific issues. As far as I'm aware, none of our teams is involved with the second type, and all of our visits are informal."

The respondent also indicated that terminology issues introduce difficulty to differentiate between therapy and entertainment, and the term "service dog" is confusing as well. 
"The general public has a lot of misunderstanding regarding the differences between service and therapy dogs. There is no national dog classification registry in the USA. We may call our dog a service dog and no-one can say otherwise. I can buy a paper for $25 \$$ on-line to certify that my dog is a therapy dog when in fact, it's not true and no service work is being done. I am not sure how to solve this issue. It causes confusion."

The results are better for professionals providing equine-assisted therapy, as there are clear boundaries between the activities included in equine-assisted therapy and equine-assisted education. There are, however, difficulties differentiating between the terms "equine-assisted therapy" and "equine-facilitated therapy".

The results indicate common understanding regarding the activities included in terms "animal-assisted therapy", "animal-assisted education", and "animal-assisted intervention", wherein the main difference concerns structured and planned work, entertainment activities, therapy goals and education through interaction with animals.

\section{Conclusions}

Animal therapy is a broad concept that includes animal-assisted therapy, animal-assisted activities and animal-assisted education. The activity of the institution, the professional and the target group determine which of the activities will be carried out in interaction with the client. Uncertainties in the use of terminology and the lack of a common viewpoint cause difficulties in identifying the content of work with therapy animals.

Different animal species should undergo a variety of tests, which may be recognised at national level.

Although interviewees had difficulty both explaining the different terms of animalassisted intervention and identifying what activities each of the terms include when integrating therapy animals into professional activities. A clear distinction was made between the institutions that integrate therapy animals in their activities and define the purpose and content of their involvement accordingly. This indicates the autonomy of the institution to define the content, regularity and professionals involved in the activities, as well as to identify the goal they want to achieve through integration of a therapy animal into the activities of the institution.

The institution has the autonomy to identify the criteria, guidelines and rules to be met for the therapy animal to be present in that institution. Depending on the institution, these rules may be more flexible or specific (e.g. in a nursing home and hospital). Consideration should be given to the activities of national and international non-governmental institutions specialising in certification and registration of therapy animals, which may help to develop and define requirements at institutional and professional level in order to develop work with therapy animals.

Prior to integration of a therapy animal into professional work with a specific client/patient, it should be assessed whether there are any restrictive factors for not doing so, for example, cultural differences, specific understanding of the animal species, its importance and interaction with humans, negative experience in interaction with animals, and allergies, etc.

\section{References}

[1] M. DeMello, Animals and Society: An Introduction to Human-Animal Studies (Columbia University Press, 2012), p. 488

[2] A.H. Fine, Animals as Social Support, Benefits of AAT. Handbook on animal-assisted therapy: theoretical foundations and guidelines for practice (Elsevier/Academic Press, 2006) 
[3] C.L. Norton, Innovative Interventions in Child and Adolescent Mental Health (Routledge, 2010), p. 196

[4] G.F. Melson, P.H. Kahn Jr., A. Beck, B. Friedman, T. Roberts, E. Garrett, B.T. Gill, J. Appl. Dev. Psychol. 30, 92-102 (2009). Available: http://www.vsdesign. org/publications/pdf/Melson2-2009-Robot-Dogs.pdf

[5] R. Ros, M. Nalin, R. Wood, P. Baxter, R. Looije, Y. Demiris, Child-Robot Interaction in the Wild: Advice to the Aspiring Experimenter, Proceedings of the 13th International Conference on Multimodal Interfaces (2011), pp. 335-342. Available: https://doi.org/10.1145/2070481.2070545

[6] M.R. Banks, L.M. Willoughby, W.A. Banks, J. Amer. Med. Directors Assoc. 9(3), 173-177, (2008). Available: https://www.clinicalkey.com/\#!/content/ playContent/1-s2.0-S1525861007005166

[7] C.A. Simmons, P. Lehmann, J. Interpers. Violence 22(9), 1211-1222 (2007) Available: http://jiv. sagepub.com. www. libproxy. wvu.edu/content/22/9/1211. full. pdf + html

[8] S.J. Thompson, H. McManus, J. Lantry, L. Windsor, P. Flynn, Evaluat. Prog. Plan. 29(1), 29, 34-43 (2006) Available: http://ac.els-cdn.com.www.libproxy. wvu .edu/S0149718905000844/1-s2.0-S0149718905000844-main.pdf?_tid= dcabfc1a-b2ca-11e6-a017-00000aacb35e\&acdnat $=1480049683 \_01 \mathrm{~cd} 1434 \mathrm{bb}$ a9f874e2e5ece214c94777

[9] J.J. Ensminger, Service and Therapy Dogs in American Society: Science, Law and the Evolution of Canine Caregivers (Charles C Thomas Publisher, 2010), p. 325

[10] P.D. McCardle, How Animals Affect Us: Examining the Influence of Human-Animal Interaction on Child Development and Human Health, (Washington DC, American Psychological Association, 2011)

[11] A.M. Dawn, Therapy Dogs in Cancer Care: A Valuable Complementary Treatment, (New York, Springer, 2012)

[12] R. Lindquist, M. Snyder, M.F. Tracy, Complementary \& Alternative Therapies in Nursing (Springer Publishing Company, 2011)

[13] P. Mongillo, E. Pitteri, S. Adamelli, S. Bonichini, L. Farina, L. Marinelli, J. Veterin. Behav. 10(2), 103-110 (2015) Available: http://ac.els-cdn.com/ S1558787814002287/1-s2.0-S1558787814002287-main.pdf?_tid=554657d8aa83-11e6-ba78-00000aab0f6b\&acdnat=1479139353_faeb3f79753adf0bc9f $5 f 101 \mathrm{bc} 471 \mathrm{a} 6 \mathrm{f}$

[14] Canine Good Citizent. What is CGC? Accessed: https : //www . akc.org/productsservices/training-programs/canine-good-citizen/

[15] Alliance of Therapy Dogs. Accessed: https://www.therapydogs.com/ alliance-therapy-dogs/ 